\title{
Treatment of internal carotid artery dissection with Willis covered stent: A case report of recurrent limb weakness and no response to medical therapy
}

\author{
XUELI CAI $^{1 *}$, JIANHONG GUAN $^{2 *}$, SHAOJUN REN $^{1}$, YIXIN WEI $^{1}$, XIAO PENG $^{1}$, WEIWEN QIU $^{1}$ and JUN CHEN ${ }^{3}$ \\ ${ }^{1}$ Department of Neurology, Lishui Central Hospital, Lishui, Zhejiang 323000; ${ }^{2}$ Department of Neurology, \\ Yanbian University Hospital, Yanbian, Jilin 133000; ${ }^{3}$ Department of Tumor Radiotherapy and Chemotherapy, \\ Ningbo Yinzhou People's Hospital, Ningbo, Zhejiang 315040, P.R. China
}

Received May 20, 2015; Accepted January 15, 2016

DOI: 10.3892/etm.2016.3112

\begin{abstract}
Internal carotid artery dissection (ICAD) is a major cause of ischemic stroke in young and middle-aged patients. Patients may be asymptomatic or present with symptoms ranging from headache and neck pain to severe cerebral ischemic events. Conventional treatment is medical anticlotting therapy or involves the use of interventional tools, such as endovascular treatment. Anticoagulation or antiplatelet therapy are the primary treatment modalities used to prevent thromboembolic complications from arterial dissections, however, they are unsuitable in certain cases of dissecting aneurysms. In the current study reports the case of a 52-year-old male patient presenting with the primary complaint of left limb weakness. Computed tomography angiography revealed a right ICAD located in the oropharyngeal segment. Subsequently, digital subtraction angiography was performed to assess the oropharyngeal segment. Antithrombotic therapy resulted in no improvement; therefore, endovascular treatment with the insertion of a Willis covered stent was performed, resulting in an improved outcome.
\end{abstract}

\section{Introduction}

Internal carotid artery dissection (ICAD) is a significant cause of ischemic stroke, and accounts for $\leq 25 \%$ of ischemic

Correspondence to: Mr. Weiwen Qiu, Department of Neurology, Lishui Central Hospital, 289 Kuocang Road, Lishui, Zhejiang 323000, P.R. China

E-mail:weiwenqiu15@163.com

Mr. Jun Chen, Department of Tumor Radiotherapy and Chemotherapy, Ningbo Yinzhou People's Hospital, 251 Baizhang East Road, Ningbo, Zhejiang 315040, P.R. China

E-mail: junchen72@163.com

*Contributed equally

Key words: internal carotid artery dissection, Willis covered stent, recurrent limb weakness strokes in young and middle-aged patients (1). The majority $(70 \%)$ of patients are reported to be $<50$ years old, with a slight predominance of male patients $(2,3)$. Common symptoms of carotid artery dissection include pain, partial Horner syndrome, cranial nerve palsies and cerebral ischemia (4). Obtaining a clinical diagnosis can be challenging; however, with an increased awareness and the use of modern instruments of investigation, such as magnetic resonance imaging and computed tomography angiography, the diagnosis of carotid dissection has become increasingly sophisticated (5). The prognosis of patients is variable; the disease may be asymptomatic, but may cause profound neurological deficits or even mortality (6). Medical treatment with anticoagulation or antiplatelet therapy, such as warfarin, heparin, rivaroxaban and aspirin, is the predominant treatment modality employed to prevent thromboembolic complications from arterial dissections (1); however, they may be unsuitable in certain cases of dissecting aneurysms $(7,8)$. The placement of a covered stent has emerged as a promising therapeutic strategy for the treatment of aneurysms (9). In particular, minimally invasive endovascular treatment with a Willis covered stent has been proposed as a safe, durable and curative therapy for selected intracranial aneurysms $(10,11)$. However, to the best of our knowledge, the use of a stent graft specifically designed for intracranial vasculature, such as the Willis expanded polytetrafluoroethylene-covered stent, has yet to be explored in the treatment of patients with ICAD. The present study reports a case of ICAD with the major complaint of recurrent left limb weakness. As antithrombotic therapy resulted in no improvement, Willis covered stent treatment was applied and resulted in an improved outcome.

\section{Case report}

The present study was approved by the ethics committee of Lishui Central Hospital (Lishui, China). A 52-year-old male patient presented to our hospital on October 2, 2014, with the primary complaint of recurrent left limb weakness for 2 months. The patient had a history of hypertension for 5 years and was diagnosed with transient cerebral ischemia in Qingtian People's Hospital (Lishui, China) in September 2014. 

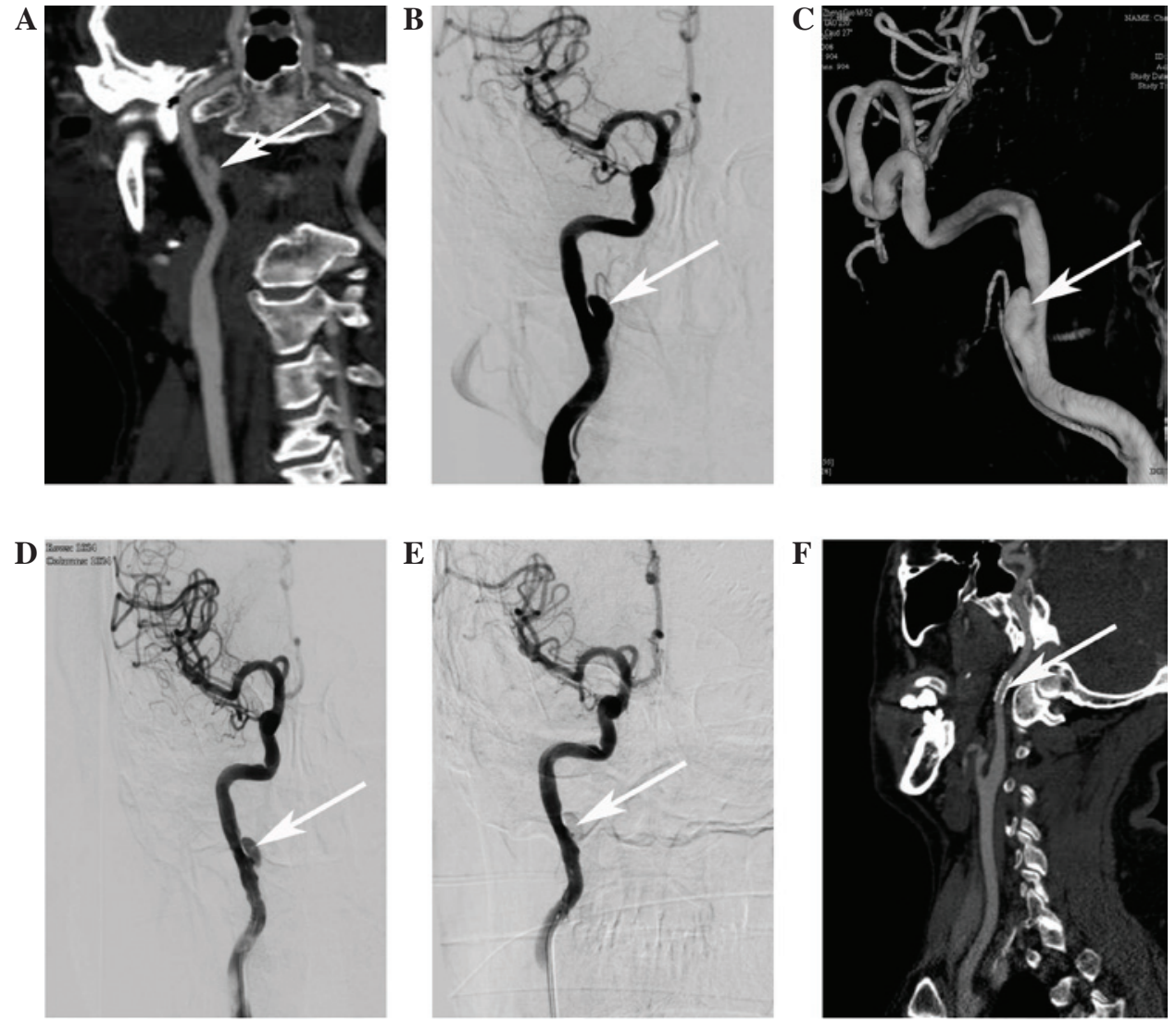

Figure 1. Angiograms of a 52-year-old man presenting with internal carotid artery dissection (ICAD) prior to and after endovascular reconstruction with a Willis covered stent (white arrow). (A) Computed tomography angiography (CTA) revealed a right ICAD located in the oropharyngeal segment. (B and C) Digital subtraction angiography (DSA) further assessed the oropharyngeal segment of the right ICAD. (D) DSA immediately after Willis covered stent placement revealed minimal endoleak into the aneurysm. (E) Repeated DSA following re-inflation of the Willis covered stent indicated the absence of the residual cavity of the aneurysm. (F) Follow-up CTA 3 months after the procedure revealed complete exclusion of the aneurysm and good patency of the parent artery.

The patient was administered an oral intensive therapy for 12 days in Qingtian People's Hospital, including aspirin [100 mg once daily (qd); Bayer, Leverkusen, Germany], clopidogrel (75 mg qd; Plavix; Sanofi S.A. Paris, France) and atorvastatin (40 mg every night; Pfizer, Inc., Shanghai, China), the patient's recurrent left limb weakness persisted. Upon presentation to our hospital, blood pressure was recorded as $186 / 101 \mathrm{mmHg}$. This was treated with amlodipine (5 mg/day; China Resources Saike Pharmaceutical Co., Ltd., Beijing, China) and benazepril (10 mg/day; Shenzen Xinlitai Pharmaceutical Industry, Ltd., Shenzen, China). The patient had not experienced any recent injury or infections and revealed no other symptoms, such as pain or dysphasia. Physical examination revealed no abnormalities of the nervous system. Furthermore, laboratory tests and head magnetic resonance imaging revealed no further abnormalities. The limb muscle strength at the time of presentation was grade I-II (12). Computed tomography angiography (CTA) revealed a right ICAD located in the oropharyngeal segment (Fig. 1A). Digital subtraction angiography (DSA) was performed to further assess the oropharyngeal segment of the right ICAD (Fig. 1B and C). The aneurysm measured $10 \mathrm{~mm}$ in length and $5 \mathrm{~mm}$ in diameter, and was accompanied by a narrowed internal carotid artery (ICA) lumen. Due to the risk of rupture and bleeding with dissecting aneurysms or cerebral infarction by arterial occlusion, in addition to the poor efficacy of chemotherapy $(13,14)$, endovascular interventional therapy was performed using a Willis covered stent (MicroPort Medical Co., Ltd., Shanghai, China).

An endovascular procedure was performed under regional anesthesia and full heparinization (6,000 units; intravenous injection $5 \mathrm{~min}$ prior to surgery) of the patient. A 6-French Envoy guiding catheter (Codman \& Shurtleff, Inc., Raynham, MA, USA) was placed in the proximal cervical segment of the right ICA and a loach guidewire (Stryker Neurovascular, West Valley City, UT, USA) was navigated into a distal branch of the ICA. Using roadmap guidance, the Willis covered stent (4x16 mm; MicroPort Medical Co., Ltd.) was navigated over the micro guidewire and bridged the aneurysm orifice. DSA was performed to confirm the positioning. The stent was then deployed with 10 atm pressure. DSA was performed immediately after deflation of the balloon to confirm the correct placement of the stent and minimal endoleakage was observed (Fig. 1D). The graft was re-inflated with 12 atm pressure to cover the orifice of the dissecting aneurysm and to completely eliminate the endoleakage. Repeated DSAs were performed immediately after the procedure to confirm the disappearance of the residual cavity of the aneurysm (Fig. 1E). During and after surgery, the patient did not complain of discomfort and no neurological deficits or complications were observed. Low molecular weight heparin (4,000 units; Jiangsu Wanbang Biochemical Pharmaceuticals Co., Ltd., Xuzhou, China) was subcutaneously injected $4 \mathrm{~h}$ after the surgery and every $12 \mathrm{~h}$ thereafter for 3 days, and the patient was then prescribed with 
oral clopidogrel (75 mg/day) and aspirin (100 mg/day) for 6 months. Follow-up CTA obtained 3 months after the procedure revealed complete exclusion of the aneurysm and patency of the parental artery (Fig. 1F).

\section{Discussion}

ICAD is most frequently observed in young to middle-aged patients, and is an important cause of ischemic stroke. It may occur spontaneously or have a traumatic etiology. The reported incidence of symptomatic spontaneous dissection is $\sim 3 / 100,000$ per year (15). Systemic vascular disorders or vasculitides, infections, smoking, diabetes mellitus and hypertension have been reported as intrinsic etiological factors (16). As aforementioned, there is a male predominance regarding ICAD incidence rates (17). Furthermore, the clinical presentation of ICAD is variable: Patients may be asymptomatic or present with various symptoms, including pain, tinnitus, Horner's syndrome, contralateral limb weakness, and retinal or cerebral ischemic symptoms (16). The diagnosis of ICAD is generally made by CTA or magnetic resonance angiography, in combination with confirmation by DSA (18). The current study reported the case of 52-year-old male patient who presented with the primary symptom of left limb weakness and no typical clinical signs, such as pain, carotid territory ischemia or Horner syndrome. However, the patient had a history of hypertension. The ICAD was restricted to the oropharyngeal segment of the right ICA, as demonstrated by CTA and DSA.

Current therapeutic strategies for ICAD include medical anticlotting therapy, endovascular treatment or surgery; however, the selection of an appropriate therapy remains controversial (4). Anticoagulant therapy in the acute stage is justified based on the presumed embolic mechanism, and antiplatelet agents, including aspirin or clopidogrel, have also been used for residual luminal irregularity or in cases of ICAD without ischemic symptoms (2). Endovascular treatments, including angioplasty or stenting, may be considered if the patient has persistent high grade stenosis despite adequate anticoagulation and a persisting or newly developed aneurysm of the dissected internal carotid, or is contraindicated for anticoagulation therapy (3). Willis covered stent is an expandable balloon-covered stent specifically developed for intracranial vessels (19). It has been previously used for treatment of patients with intracranial aneurysms, internal carotid artery aneurysms and pseudoaneurysms, and has been suggested to be a safe, durable and efficacious option $(20,21)$. However, endoleakage is a frequent problem following initial implantation (10), and in the case of a tortuous parent artery or potential side branch coverage, using a Willis covered stent is not recommended (22). In the present case, medical therapy with aspirin, clopidogrel and atorvastatin resulted in no obvious improvement of the recurrent contralateral limb weakness, therefore, endovascular reconstruction of the right ICAD was performed using a Willis covered stent; expandable balloon-covered stent specifically developed for intracranial vessels (14). The stent was successfully deployed and anchored at the targeted vessel segment and the performance of the patient was markedly improved thereafter. Antiplatelet therapy was reported to be important following the use of the device (23). Thus, the patient received an antiplatelet protocol and no significant in-stent stenosis was identified on the follow-up DSA. However, endoleakage following placement of the covered stent is a risk that needs to be carefully monitored.

In conclusion, present study revealed the significant role of interventional procedures using the Willis covered stent in the treatment of unilateral ICAD when medical therapy has proven ineffectual.

\section{References}

1. Vicenzini E, Ricciardi MC, Sirimarco G, Di Piero V and Lenzi GL: Bilateral spontaneous internal carotid artery dissection with both early and very late recanalization: A case report. J Clin Ultrasound 39: 48-53, 2011.

2. Soong B, Chan $\mathrm{H}$ and $\mathrm{Ng} \mathrm{F}$ : A case of young man with stroke caused by internal carotid artery dissection. Hong Kong J Emerg Med 19: 49, 2012.

3. Kim Y-K and Schulman S: Cervical artery dissection: Pathology, epidemiology and management. Thromb Res 123: 810-821, 2009.

4. Patel RR, Adam R, Maldjian C,Lincoln CM, Yuen A and Arneja A: Cervical carotid artery dissection: Current review of diagnosis and treatment. Cardiol Rev 20: 145-152, 2012.

5. Mohan IV: Current optimal assessment and management of carotid and vertebral spontaneous and traumatic dissection. Angiology 65 274-283, 2014.

6. Bayır A, Aydoğdu Kıreşi D, Söylemez A and Demirci O: Cerebral infarction caused by traumatic carotid artery dissection. Ulus Travma Acil Cerrahi Derg 18: 347-350, 2012.

7. Nakiri GS, Al-Khawaldeh M, Parente B, Kessler I, Gory B, Riva R and Mounayer C: Treatment of ruptured intra-cranial internal carotid artery dissection using a flow-diverter stent. J Neuroradiol 39: 271-275, 2012.

8. Feugier P, Vulliez A, Bina N, Floccard B and Allaouchiche B: Urgent endovascular covered-stent treatment of internal carotid artery injury caused by a gunshot. Eur J Vasc Endovasc Surg 34: 663-665, 2007.

9. Zhu YQ, Li MH, Lin F, Song DL, Tan HQ, Gu BX, Zhang HQ, Leng B and Zhang PL: Frequency and predictors of endoleaks and long-term patency after covered stent placement for the treatment of intracranial aneurysms: A prospective, non-randomised multicentre experience. Eur Radiol 23: 287-297, 2013.

10. Tan HQ, Li MH, Li YD, Fang C, Wang JB, Wang W, Wang J, Zhang PL and Zhu YQ: Endovascular reconstruction with the Willis covered stent for the treatment of large or giant intracranial aneurysms. Cerebrovasc Dis 31: 154-162, 2011.

11. Zhu YQ, Gu BX, Li MH, Wang W, Cheng YS, Tan HQ, Wang JB, Zhang PL and Ma LT: Safety, feasibility, and mid-term follow-up of Willis stent graft placement in the treatment of symptomatic complicated intra-or extra-cranial aneurysms: A multicenter experience. Minim Invasive Ther Allied Technol 19: 320-328, 2010.

12. Ping JJ, Di CS and Ying CL (eds): Neurology. 7th edition. People's Medical Publishing House, Shelton, CT, 2013.

13. Bogousslavsky J and Pierre P: Ischemic stroke in patients under age 45. Neurol Clin 10: 113-124, 1992.

14. Touzé E, Gauvrit JY, Moulin T, Meder JF, Bracard S, Mas JL; Multicenter Survey on Natural History of Cervical Artery Dissection: Risk of stroke and recurrent dissection after a cervical artery dissection: A multicenter study. Neurology 61: 1347-1351, 2003.

15. Debette S and Leys D: Cervical-artery dissections: Predisposing factors, diagnosis, and outcome. Lancet Neurol 8: 668-678, 2009.

16. Salvati B, Tesori MC, Lombardo F, Donello C, Lange KJ and Capoano R: Surgical treatment of spontaneous common carotid dissection: A case report. Ann Ital Chir 85 (ePub): 85, 2014.

17. Metso AJ, Metso TM, Debette S, Dallongeville J, Lyrer PA, Pezzini A, Lichy C, Kloss M, Brandt T, Touzé E et al: CADISP Group: Gender and cervical artery dissection. Eur J Neurol 19: 594-602, 2012.

18. Hassan AE, Zacharatos H, Rodriguez GJ, Suri MF, Tariq N, Vazquez G, Tummala RP and Qureshi AI: Long-term clinical and angiographic outcomes in patients with spontaneous cervico-cranial arterial dissections treated with stent placement. J Neuroimaging 22: 384-393, 2012.

19. Tan HQ, Li MH, Zhang PL, Li YD, Wang JB, Zhu YQ and Wang W: Reconstructive endovascular treatment of intracranial aneurysms with the Willis covered stent: Medium-term clinical and angiographic follow-up. J Neurosurg 114: 1014-1020, 2011. 
20. Wang W, Li MH, Li YD, Gu BX, Wang J, Zhang PL and Li M: Treatment of traumatic internal carotid artery pseudoaneurysms with the Willis covered stent: A prospective study. J Trauma 70: 816-822, 2011.

21. Zhu YQ, Li MH, Fang C, et al.: Application of the Willis covered stent in the treatment of aneurysm in the cisternal segment of the internal carotid artery: a pilot comparative study with midterm follow-up. J. Endovasc. Ther. 17: 55-65, 2010 .
22. Li M-H, Gao B-L, Wang Y-L, Fang C and Li Y-D: Management of pseudoaneurysms in the intracranial segment of the internal carotid artery with covered stents specially designed for use in the intracranial vasculature: Technical notes. Neuroradiology 48: 841-846, 2006.

23. Wang JB, Li MH, Fang C, Wang W, Cheng YS, Zhang PL, Du ZY and Wang J: Endovascular treatment of giant intracranial aneurysms with willis covered stents: Technical case report. Neurosurgery 62: E1176-E1177, 2008. 